\title{
Background of Recommendation IX
}

\section{McCarthy}

U.S. Naval Observatory, Washington, 20392

Due to weather-related travel delays Dr. McCarthy was unable to present his scheduled paper. In his absence, J.A. Hughes gave a short talk indicating that Recommendation IX had received essentially universal approval at and since the Virginia Beach Colloquium, and that it would undoubtedly be approved by the Joint Commission Meeting as presently written, and then submitted as a Commission Resolution.

\section{DISCUSSION}

\section{Kinoshita:}

I would like a brief comment from the view point of the IERS. As stated in the second paragraph of Recommendation IX, the IERS has published or computed the so-called celestial pole off-set, which means the excess angle from the adopted 1980 nutation series. And the importance of the nutation has, I think, two implications, one is geophysical of course, and the other is the implication for astrometry or astronomy. And in this text it is written that only nutation is included, but the celestial pole off-set which IERS has published includes both precession and nutation. So at the present stage it is not very efficient or very easy to separate the precession and nutation based on, for example, the 11 years of VLBI data, and if we include the lunar data, still it's not easy. From the geophysical point of view it is still difficult to model the dissipation processes which produce the so-called out of phase components. So from the practical points of view of IERS performance, we also decided to keep the present nutation series as it is now. And after a few years, I hope, when a complete geophysical model is established, then it may be applied, but at the present stage I think the series should be kept as it is now. The erroneous precession and nutation of course affect the compilation of the radio reference system which has been kept in the IERS/VLBI community. But the differences of the systems of individual radio reference catalogs are adjusted by checking, by comparing the individual catalogs, and calculating the so-called rotation angles, and also the effects of the erroneous nutation effects. The Earth's rotation parameters are also adjusted by the comparison of the individual series of VLBI. So, the IERS would like to take these procedures to provide a so-called unique IERS celestial reference frame, based on the present nutation series. 